\title{
Simulation of multi-platform LiDAR for assessing total leaf area
}

\section{in tree crowns}

Ting Yun ${ }^{1,2,{ }^{\dagger}}$, Lin $\mathrm{Cao}^{2,+}$, Feng An ${ }^{3}$, Bangqian Chen ${ }^{3}$, Lianfeng Xue ${ }^{1 *}$, Weizheng $\mathrm{Li}^{4}$, Sylvain Pincebourde ${ }^{5}$, Martin J. Smith ${ }^{6}$ and Markus P. Eichhorn ${ }^{7,8}$

${ }^{1}$ School of Information Science and Technology, Nanjing Forestry University, Nanjing 210037, China

${ }^{2}$ Co-Innovation Centre for Sustainable Forestry in Southern China, Nanjing Forestry University, Nanjing 210037, China

${ }^{3}$ Danzhou Investigation and Experiment Station of Tropical Crops, Ministry of Agriculture, Rubber Research Institute, Chinese Academy of Tropical Agricultural Sciences, Danzhou 571737, China

${ }^{4}$ Advanced Analysis and Testing Centre, Nanjing Forestry University, Nanjing 210037, China

${ }^{5}$ Institut de Recherche sur la Biologie de I'Insecte (IRBI), UMR 7261, CNRS/Universite de Tours, 37200 Tours, France

${ }^{6}$ Faculty of Engineering, The University of Nottingham, University Park, Nottingham, NG7 2RD, United Kingdom

${ }^{7}$ School of Biological, Earth and Environmental Sciences, University College Cork, Distillery Fields, North Mall, Cork, T23 N73K, Ireland

${ }^{8}$ Environmental Research Institute, University College Cork, Lee Road, Cork, T23 XE10, Ireland

${ }^{*}$ Corresponding author: Lianfeng Xue (xuelianfeng@njfu.edu.cn)

† These authors contributed equally to this work. 
Abstract: LiDAR (Light Detection and Ranging) technology has been increasingly implemented to assess the biophysical attributes of forest canopies. However, LiDAR-based estimation of tree biophysical attributes remains difficult mainly due to the occlusion of vegetative elements in multi-layered tree crowns. In this study, we developed a new algorithm along with a multiplescan methodology to analyse the impact of occlusion on LiDAR-based estimates of tree leaf area. We reconstructed five virtual tree models using a computer graphic-based approach based on in situ measurements from multiple tree crowns, for which the position, size, orientation and area of all leaves were measured. Multi-platform LiDAR simulations were performed on these 3D tree models through a point-line intersection algorithm. An approach based on the Delaunay triangulation algorithm with automatic adaptive threshold selection was proposed to construct the scanned leaf surface from the simulated discrete LiDAR point clouds. In addition, the leaf area covered by laser beams in each layer was assessed in combination with the ratio and number of the scanned points. Quantitative comparisons of LiDAR scanning for the occlusion effects among various scanning approaches, including fixed-position scanning, multiple terrestrial LiDAR scanning and airborne-terrestrial LiDAR cross-scanning, were assessed on different target trees. The results showed that one simulated terrestrial LiDAR scan alongside the model tree captured only $25-38 \%$ of the leaf area of the tree crown. When scanned data were acquired from three simulated terrestrial LiDAR scans around one tree, the accuracy of the leaf area recovery rate reached $60-73 \%$ depending on the leaf area index, tree crown volume and leaf area density. When a supplementary airborne LiDAR scanning was included, occlusion was reduced and the leaf area recovery rate increased to $72-90 \%$. Our study provides an approach for the measurement of total leaf area in tree crowns from simulated multi-platform LiDAR data and enables a quantitative assessment of occlusion metrics for various tree crown attributes under different scanning strategies.

Keywords: Laser scanning; LiDAR; Leaf area; Occlusion effect; Computer graphics. 


\section{Introduction}

Leaves are the dominant exchange surfaces of plants. The leaf area of each tree is a primary physiological and biogeochemical determinant of its overall rates of photosynthesis, carbon uptake and transpiration (Boegh et al., 2002). Variation in leaf area among trees substantially alters the output of ecological models (Chen et al., 2005). Therefore, accurate estimates of the leaf area of tree crowns are required to understand the ecosystem functions provided by trees. However, leaf area remains a difficult parameter to measure, especially in complex tree crowns such as forests. There are few reliable non-destructive methods for obtaining high-quality measurements, whereas destructive methods are too time consuming to be widely applied.

LiDAR (Light Detection and Ranging) offers an opportunity to conduct measurements of vegetation from different viewpoints at finer resolutions than have previously been available. Commercially available equipment, such as terrestrial (Lu et al., 2014), ground vehicle-loaded (Asvadi et al., 2016) and aerial laser scanning (Liu et al., 2017) devices, can rapidly generate point cloud data that can be used to reconstruct the 3D structure of vegetation. The fine characterization of morphological features of broad-leaf trees acquired via bottom-up or topdown scanning allows tree crowns, trunks, branches, twigs and often even leaves, to be easily distinguished through visual inspection. These high-resolution structural measurements provide an exciting opportunity to directly capture leaf area.

A number of approaches were developed to process LiDAR point clouds and estimate the leaf area of tree crowns in recent years. Based on the gap fraction theory (Nilson, 1971) and probabilistic approaches, many theoretical models were developed, including (i) a maximum likelihood estimator combined with a Poisson gap model to estimate gap fraction and leaf area index (Zhao et al., 2015), (ii) calculation of the contact frequency within each voxel to estimate gap fraction and correct for the influence of occlusion effects on the leaf area estimate (Li et al., 2017), (iii) the use of multi-return LiDAR data to evaluate laser penetration metrics through the tree crowns and to compute the gap fraction inversion (Alonzo et al., 2015), (iv) determination of the canopy extinction coefficient of foliage elements by considering both inclination and azimuth 
angles of leaves from different laser scanning data (Ma et al., 2017) and (v) the development of a 3D modelling framework of forest stands to quantify within-crown clumping factors and to evaluate the gap fraction of tree crowns (Woodgate et al., 2015). These previous studies were based on optics theory, which considers laser hits to be a complete sample. The number of laser returns within a given zenith angle range is used to estimate the gap probability within the tree crown, from which the leaf area index (LAI) can be computed.

Despite the recognized advantages of LiDAR techniques, the analysis of complex point clouds and the extraction of metrics relevant to ecology and environmental science still face practical challenges. A persistent problem with the direct measurement of LAI is occlusion in dense tree crowns caused by multiple overlapping leaf surfaces and branches. Thus, any method should compensate for leaves that are not visible from the perspective of the laser position (Côté et al., 2012; Van der Zande et al., 2011). When developing an algorithm to retrieve leaf area estimates, it is essential to incorporate measures of uncertainty related to the presently unquantifiable issue of signal occlusion. The representation of a scanned tree as a discrete set of points is also difficult to relate directly to the leaf surface area (Yun et al., 2016). The spatial resolution of scanned points is inversely proportional to the acquisition distance (Méndez et al., 2013; Pesci et al., 2011), which means that a higher density of scanned points will be acquired when foliage is closer to the scanner, leading to a non-uniform resolution of canopy structures. During the scanning process, wind blowing on the leaves and the intrinsic properties of the sensors cause noise in the scanned data, which complicates the characterization of tree crown attributes.

Computer graphics of geometric data is another approach used for performing feature calculations and rendering 3D models, which are used to accurately describe complex, non-rigid and irregular objects, such as bunch grasses, trees and forests. Computer graphical methods combined with computer vision and image processing methods have been applied to retrieve forest properties and extract the key parameters from the scanned data. These methods include irradiation geometry to analyse the radiation regime in a forest canopy and measurement of the 
gap fraction of forest plots (Van Leeuwen et al., 2013), 3D triangulation to produce a geometrically explicit description of a forest canopy from airborne point cloud data (Vauhkonen et al., 2016), point cloud-based triangulation for laser-leaf intersection points for the rapid measurement of the 3D distribution of leaf orientation and leaf angle probability density (Bailey and Mahaffee, 2017), graphic projection strategies related to the tree point cloud to deduce tree row scanned volume and leaf area (Sanz et al., 2018), individual leaf segmentation and 3D leaf surface reconstruction to characterize the morphological properties of agricultural plants (Chaivivatrakul et al., 2014), and leaf area and height estimation using geometric features from sparse 3D points generated from stereovision models (Lati et al., 2013).

The main objective of the present study was to develop a computer graphic-based methodology to quantify leaf area and the occlusion effect for different LiDAR scanning patterns. This aim was achieved by developing a computer graphics algorithm to extract the total leaf area covered by simulated multi-platform LiDAR point clouds and by implementing a computer reconstruction technique to validate the results. The specific objectives were: 1 ) to generate 3D virtual tree models obtained from field measurements and to design a simulation algorithm to conduct virtual laser scanning of the trees; 2) to develop a computer graphics-based approach for calculating total leaf area covered by laser beams from discrete LiDAR point clouds using Delaunay triangulation with an adaptively chosen threshold; and 3) to assess different stratified strategies of scanned points combined with the defined ratio of point number per unit leaf area for evaluating the occlusion effects on the leaf area retrieval corresponding to different scanning patterns (i.e., fixed-position scanning, multiple terrestrial scanning combination and aerialterrestrial cross-scanning). Through this we provide and validate a novel method for direct leaf area estimates based on the point cloud coverage under a range of scanning scenarios. 


\section{Materials and Methods}

\subsection{Field data}

Five datasets comprising one mango tree (Mangifera indica L.), two rubber trees (Hevea brasiliensis Müll.Arg.), one walnut tree (Juglans X intermedia.) and one apple tree (Malus communis Desf.) were obtained from Sinoquet et al. (2009). All samples were isolated trees with no crown contact. The 3-year-old walnut tree was grown in a research field at the INRA Research Centre in Clermont-Ferrand, France. The two-year-old mango tree was grown in an orchard in Ban Bung, near Chonburi, Thailand. The smaller 2-year-old rubber tree was grown in the garden of the Department of Agronomy Kasetsart University, Bangkok, Thailand. The larger 5-year-old rubber tree was grown at the Suwan Wajokkasikit Field Crops Research Station, Pak Chong, Thailand. The 20-year-old apple tree was located on a private property in Vouvray, near Tours, France. The spatial coordinates and the orientation angles of every leaf, which were in the form of the midrib azimuth and inclination angles, and the rolling angle of the leaf lamina around the midrib were collected with a 3D electromagnetic digitizer (Sinoquet et al., 2009). Leaf dimensions were measured manually. The tree height varied from 1.7-10.2 m, and the number of leaves varied from $895-26,254$, yielding total leaf areas of $4.07-35.2 \mathrm{~m}^{2}$. A summary of these architectural data is provided in Table 1.

Table 1. Attributes of trees, such as height, crown diameter, number of leaves, total leaf area of the tree crown, were collected manually by (Sinoquet et al., 2009) and were used for laser scanning simulations. The tree crown volume, tree crown projection area, Leaf Area Index, average area of each leaf and sampling points on the vegetative elements were calculated from these data.

\begin{tabular}{lccccc}
\hline & Walnut & Mango & Rubber 1 & Rubber 2 & Apple \\
\hline Height $(\mathrm{m})$ & 3.9 & 1.7 & 2.2 & 10.2 & 8.3 \\
Crown diameter $(\mathrm{m})$ & 1.8 & 1.8 & 1.6 & 4.1 & 5.6 \\
\hline
\end{tabular}




\begin{tabular}{|c|c|c|c|c|c|}
\hline $\begin{array}{l}\text { Total number of } \\
\text { leaves }\end{array}$ & 1558 & 1636 & 895 & 12141 & 26254 \\
\hline $\begin{array}{l}\text { Total leaf area of the } \\
\text { tree crown }\left(\mathrm{m}^{2}\right)\end{array}$ & 7.07 & 6.56 & 4.07 & 35.10 & 37.41 \\
\hline Basal diameter $(\mathrm{cm})$ & 5.18 & 7.67 & 4.47 & 14.08 & 12.58 \\
\hline $\begin{array}{l}\text { Total number of } \\
\text { sampling points on } \\
\text { leaf surfaces }\end{array}$ & 533,007 & 535,142 & 308,077 & $2,780,905$ & $3,077,374$ \\
\hline $\begin{array}{l}\text { Total number of } \\
\text { sampling points on } \\
\text { branch surfaces }\end{array}$ & 120,490 & 198,831 & 162,325 & 596,843 & $1,077,981$ \\
\hline $\begin{array}{l}\text { Tree crown volume } \\
\left(\mathrm{m}^{3}\right)\end{array}$ & 3.20 & 1.36 & 1.32 & 23.08 & 36.95 \\
\hline $\begin{array}{l}\text { Tree crown } \\
\text { projection area }\left(\mathrm{m}^{2}\right)\end{array}$ & 1.82 & 1.43 & 1.92 & 10.58 & 12.98 \\
\hline LAI (leaf area index) & 3.89 & 4.58 & 2.12 & 3.32 & 2.88 \\
\hline $\begin{array}{l}\text { Average area of each } \\
\text { leaf }\left(\mathrm{cm}^{2}\right)\end{array}$ & 45.38 & 40.10 & 45.47 & 28.91 & 14.25 \\
\hline
\end{tabular}

\subsection{Creation of tree models}

\subsubsection{Creation of 3D tree crown models and discretization}

Three-dimensional models based on the in situ measurements were created for each selected tree. The length $l_{s}$ and width $w_{s}$ of the $s$ th leaf surface were used to approximate the leaf as a symmetrical ellipse on each side of the midrib; the initial position of each leaf surface lies on the X-Y plane, and the normal vector of the leaf surface in 3D space is $\grave{h}_{s}=(0,0,1)$. A uniform sampling strategy was adopted to obtain a set of valid points defining whole leaves. The 
sampling spacing of the points on each leaf surface was equal to the size of a grid cell $c$, set here as $0.4 \mathrm{~cm}$ (Figure 1a). Then, for every leaf in the total set of leaves, the set of points $P_{s}\left(x_{i, j}, y_{i, j}, z_{i, j}\right)$ was used to define the $i$ th row and the $j$ th column on the $s$ th leaf surface. Given the overall leaf properties assessed with rotation angles $\theta_{s}^{x}, \theta_{s}^{y}$ and $\theta_{s}^{z}$ around the $\mathrm{X}$ axis, $\mathrm{Y}$ axis and $\mathrm{Z}$ axis, respectively, and the spatial location $d_{s}\left(d_{s}^{x}, d_{s}^{y}, d_{s}^{z}\right)$, the composite transformation of the original points $P_{s}\left(x_{i, j}, y_{i, j}, z_{i, j}\right)$ in the $\mathrm{X}, \mathrm{Y}$ plane was represented as follows:

$$
P_{s}^{r}\left(x_{i, j}^{r}, y_{i, j}^{r}, z_{i, j}^{r}\right)=P_{s}\left(x_{i, j}, y_{i, j}, z_{i, j}\right) \times R_{X} \times R_{Y} \times R_{Z}
$$

New points $P_{s}^{r}\left(x_{i, j}^{r}, y_{i, j}^{r}, z_{i, j}^{r}\right)$ were thus generated to represent the position, size and shape of each individual leaf (Figure 1b). After the transformation, the new normal vector of the transformed leaf surface was adjusted to $\vec{h}_{s}^{r}=\left(h_{s, x}^{r}, h_{s, y}^{r}, h_{s, z}^{r}\right)=\vec{h}_{s} \times R_{X} \times R_{Y} \times R_{Z}$, where

$$
R_{X}=\left[\begin{array}{ccc}
1 & 0 & 0 \\
0 & \cos \left(\theta_{s}^{x}\right) & \sin \left(\theta_{s}^{x}\right) \\
0 & -\sin \left(\theta_{s}^{x}\right) & \cos \left(\theta_{s}^{x}\right)
\end{array}\right], R_{Y}=\left[\begin{array}{ccc}
\cos \left(\theta_{s}^{y}\right) & 0 & -\sin \left(\theta_{s}^{y}\right) \\
0 & 1 & 0 \\
\sin \left(\theta_{s}^{y}\right) & 0 & \cos \left(\theta_{s}^{y}\right)
\end{array}\right] \text { and } R_{Z}=\left[\begin{array}{ccc}
\cos \left(\theta_{s}^{z}\right) & \sin \left(\theta_{s}^{z}\right) & 0 \\
-\sin \left(\theta_{s}^{z}\right) & \cos \left(\theta_{s}^{z}\right) & 0 \\
0 & 0 & 1
\end{array}\right]
$$

Therefore, the equation of the $s$ th leaf surface plane can be simplified as follows:

$$
h_{s, x}^{r}\left(x^{r}-d_{s}^{x}\right)+h_{s, y}^{r}\left(y^{r}-d_{s}^{y}\right)+h_{s, z}^{r}\left(z^{r}-d_{s}^{z}\right)=0
$$

where $\left[x^{r}, y^{r}, z^{r}\right]=[x, y, z] \times R_{X} \times R_{Y} \times R_{Z}$ and $x \in\left[-l_{s} / 2, l_{s} / 2\right], \quad y \in\left[-w_{s} / 2, w_{s} / 2\right]$ and $z \in[-0,0]$. The range of $x^{r}, y^{r}$ and $z^{r}$ can be deduced from the range of variables $x, y$ and $z$ representing the initial ellipse lying on the $X-Y$ plane. All leaves together constituted a complete representation of the tree crown (Figure 1c and d). The leaf area of the tree crown was determined by summing the ellipse area of each leaf surface.

\subsubsection{Creation of tree branches and discretization}

For all target trees, prior information of each main branch obtained from successive measurements was used for tree branch construction. Branching, branch diameter, and flush 
order were given as well as the precise ranking of leaves along each branch (Sinoquet et al., 2009). A 3D pipeline mode reconstructed each branch from the proximal to distal tip until the whole branching architecture was retrieved for each target tree. Thus, the preliminary tree skeleton was delineated, and a set of generalized cylinders was assembled into a skeleton along connected vectors to form the tree branches (Figure 1e). The radii of cylinders, set as the diameter of the branches, were smaller at the upper tree crown and larger at the lower tree crown. Finally, a uniform sampling strategy with sampling spacing $c$ (equal to the size of a grid cell) was performed to transform every branch cylinder into discrete points $P_{b}\left(x_{i, j}^{b}, y_{i, j}^{b}, z_{i, j}^{b}\right)$

(Figure $1 \mathrm{f}$ and g). Finally, the tree model and corresponding discrete point set $P_{i}=\left\{x_{i, j}^{r}, y_{i, j}^{r}, z_{i, j}^{r}, x_{i, j}^{b}, y_{i, j}^{b}, z_{i, j}^{b}\right\}$ were made available for the laser scanning simulation.
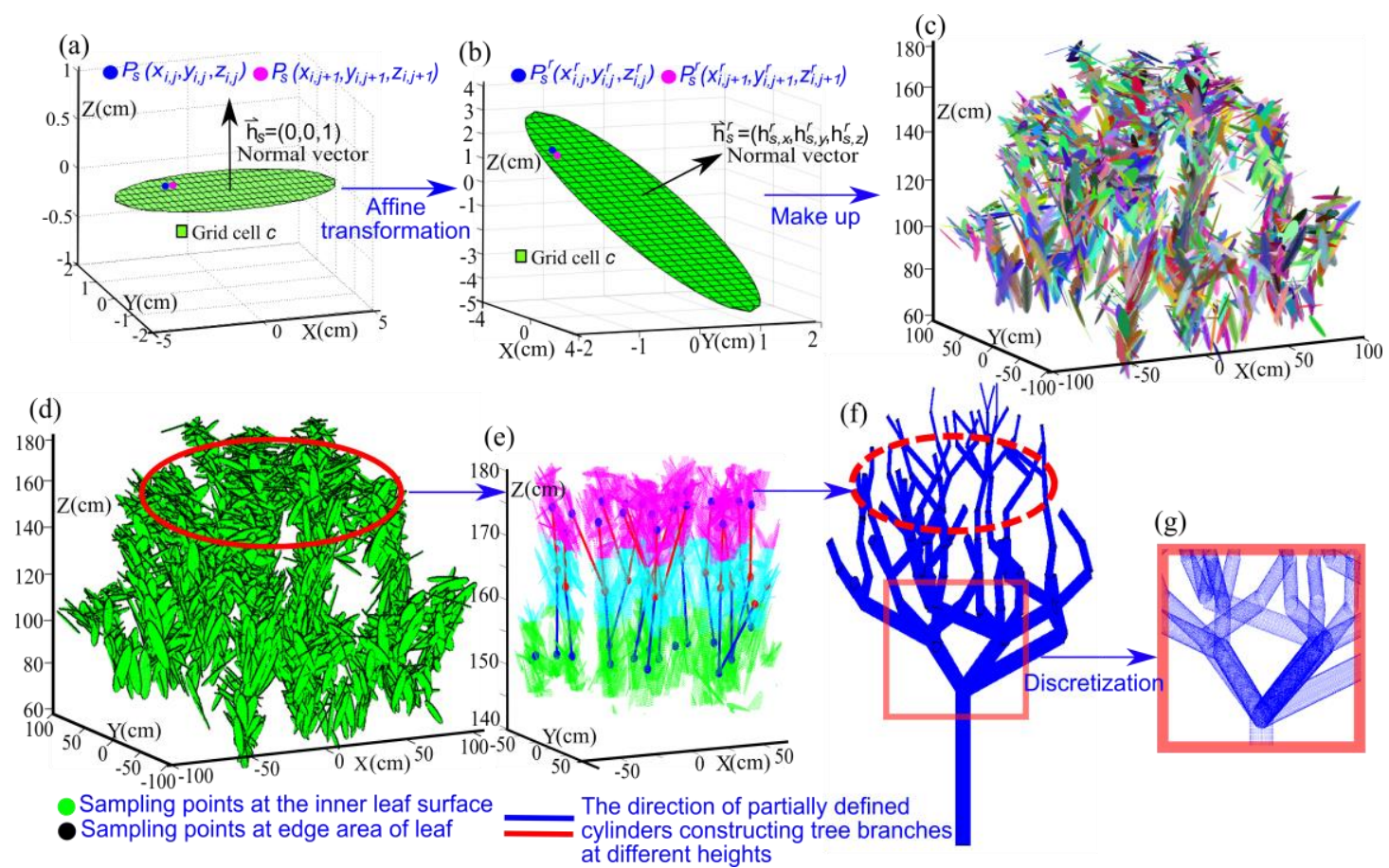

Figure 1. Schematic illustrating a tree (mango tree) reconstruction. (a) Initial sampling points of one mango leaf on a plane of the elliptical form. (b) Spatial position and inclinational and azimuthal angle of leaf attributes through affine transformation. (c) Numerous leaves with various attributes make up the three-dimensional model of the mango tree crown. (d) Sampling points covering each leaf surface make up the tree crown model; the green points represent the sampling points at the inner portion of leaf surface, and the black points represent the edges of 
each leaf. (e) The red and blue lines represent the directions of cylinders constituting the tree branches guided by field measurements. (f) Overview of the reconstructed 3D tree skeleton. (g) Close-up of partial virtual branch discretization for obtaining branch sampling points.

\subsection{Laser scanning simulation and scanned leaf area calculation}

After the tree models were reconstructed and the vegetative elements were transformed into high-density sampling points, a rapid simulation method based on the line-point intersection principle was proposed to perform various laser scanning patterns, including single or multiangle terrestrial laser scanning (TLS) and aerial laser scanning (ALS), and to acquire the corresponding scanned points of each tree model. To calculate the scanned leaf area and to evaluate the occlusion effect on laser scanning, every modelled tree crown was divided into six layers according to the scanning distance regarding various scanning patterns, and a triangulation method with automatic adaptive threshold selection was designed to transform the discrete scanned points into the leaf surface (Supplementary materials S1). The threshold was determined based on the scanning angular resolution, distance between the leaf and scanner, and angle between the incident laser beam and the normal vector of the leaf surface. Then, the ratio $\rho_{z}$ between the number of scanned points and corresponding scanned leaf area in each layer was calculated to deduce the scanned leaf area of each layer from various scanning patterns. To compare with the true leaf area, a quantitative assessment of the occlusion metric for various tree crown attributes under different scanning patterns was then performed. The flowchart of our method is shown in Figure 2, and the detailed descriptions of technical implementation are supplied in the Supplementary materials section S1.

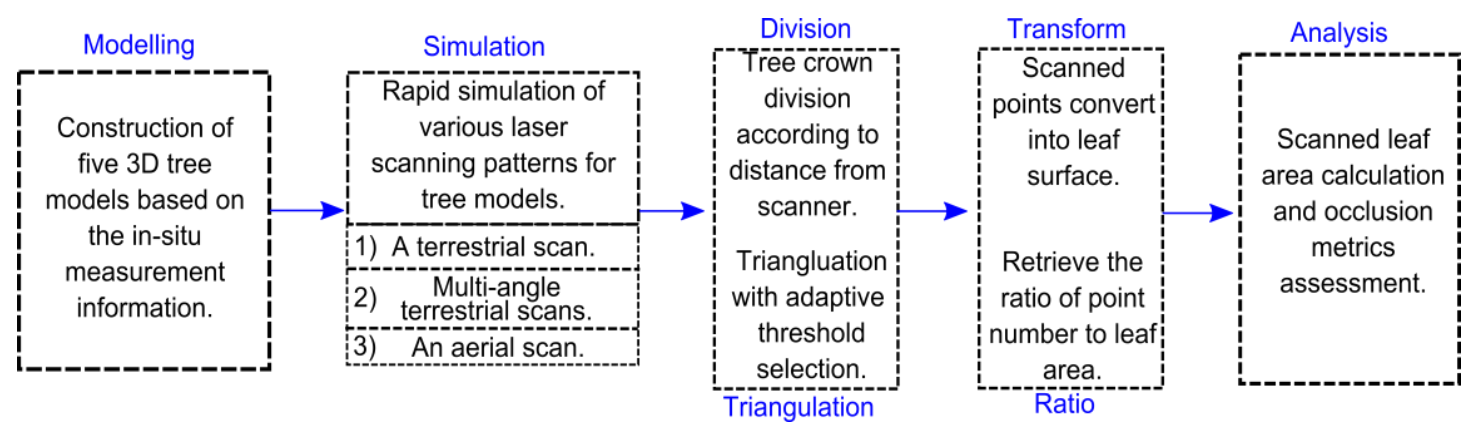

Figure 2. The flowchart illustrating the main steps of our simulation method.

Five tree crowns were taken as the subjects for scanning simulations, and virtual scans of each tree crown were conducted from either one TLS position or three TLS positions around the 
tree, with an ALS position also included. Various laser scanning simulation scenarios were conducted using our program. The scanned targets in our experiments can be either leaves alone (without branches) or leaves and branches together (with branches). Scanning simulation scenarios either with or without occlusion were also tested. In the former scenario (with the occlusion effect), only the nearest intersection point on each beam to the scanner was identified as the scanned point (i.e., the simulation based on the real process of scanned data acquisition). In the latter scenario (without the occlusion effect), every beam could pass through all vegetative elements and all intersection points between each beam and scanned vegetative elements were identified as the scanned points, i.e., a hypothesis that occlusion does not exist in the scanning process. Under the four scenarios indicated above (i.e., without branches, with branches, with occlusion and without occlusion), the leaf area retrieved in each layer from the scanned points was compared with the true leaf area directly calculated from the tree models (Section 2.2). Major parameters and the proportion of true leaf area detected are summarized in the following sections. Our laser scanning simulation method was implemented in MATLAB (The MathWorks, Inc. Natick, Massachusetts, U.S.A.), and the code can be requested from the first author. Execution of one scan simulation program takes approximately 3 minutes for a small tree and approximately 7 minutes for a large tree. The running time of our program is similar to that of a real scan accomplished using a Leica C10 scanner.

\section{Results}

\subsection{Horizontal penetration of virtual scans}

We focus first on data collected from the mango tree as an illustration of the overall pattern before comparing the results for different tree crowns. Horizontal scanning profiles reveal the degree of signal attenuation through the tree crown and, hence, the accuracy of the leaf area estimation (Figure 3). These profiles also reveal the relative degree of occlusion with one versus three scanning positions. Figure 3a shows a lateral snapshot of one scanning pattern in which different colours represent the degree to which each scanned point blocks the vegetative 
elements behind the point. The leaf elements closer to the scanner block more subsequent vegetative elements for each laser beam than the more distant leaf elements, and the missed points are further from the scanner. The number of scanned points detected per unit leaf area measured, $\rho_{z}$, and the total leaf area of each segment were calculated and are illustrated in Figure $3 \mathrm{~b}$ and $3 \mathrm{c}$ respectively. The $\rho_{z}$ estimated from the original leaf points using our Delaunay method is close to the initial sampling resolution, with discrepancies caused by the difference between true and estimated leaf area. Without occlusion, there is a decline in $\rho_{z}$ with distance that is caused by the beams spreading out in space. When perspective occlusion is included, the overall values of $\rho_{z}$ are lower due to the smaller overall number of scanned points. Moreover, when the branches are assembled into the tree crown, $\rho_{z}$ is slightly smaller than its value without branches because more leaf elements in the distant part of the tree crown from the scanner are blocked by the branches. The leaf area estimation using our method is shown in Figure 3c. From the original sampling points of leaf surfaces, the estimated results using our Delaunay triangulation method (represented by the dashed blue line with square markers in Figure 3) converge on the true leaf area, demonstrating the effectiveness of our leaf area retrieval method. When occlusion is incorporated, the reduction in leaf area retrieval highlights the effect of vegetation closer to the scanner blocking other vegetation. In the sections of tree crown closest to the scanner, almost no effect of occlusion is evident. On the distal side of the tree, only a small proportion of the total leaf area is captured. When the branches were included in the tree crown, the occlusion effect increased slightly, resulting in a decline in estimated leaf area.

Substantial improvements were realized when three TLS positions around the model tree were employed (Figure $3 \mathrm{~d}$ ). In such a set-up, the occluded points exist mainly in the tree crown centre. Thus, division of the model tree into six annulus sectors according to the distance from the tree crown centre was conducted to assess $\rho_{z}$ and total leaf area of each segment. Based on the original sampling points of the leaf surface, a distribution of $\rho_{z}$ across the tree crown was created with values similar to the initial sampling resolution (Figure 3e). In the three terrestrial 
virtual scans around the tree crown with occlusion, the overall values of $\rho_{z}$ are lower and increase with distance from the tree crown centre because the leaf elements in the tree crown centre are blocked by outer foliage elements. Thereby, $62.64 \%$ of the total leaf area of the model tree was represented by the black line in Figure 3f. When occlusion is excluded, $\rho_{z}$ reaches a theoretical maximum value in the inner portion of the tree crown, where the leaves could potentially be scanned from multiple positions. It increases again at the crown edge near the scanner. Figure $3 \mathrm{f}$ also shows that including tree branches has only a slight impact on the overall accuracy of the total leaf area assessment.
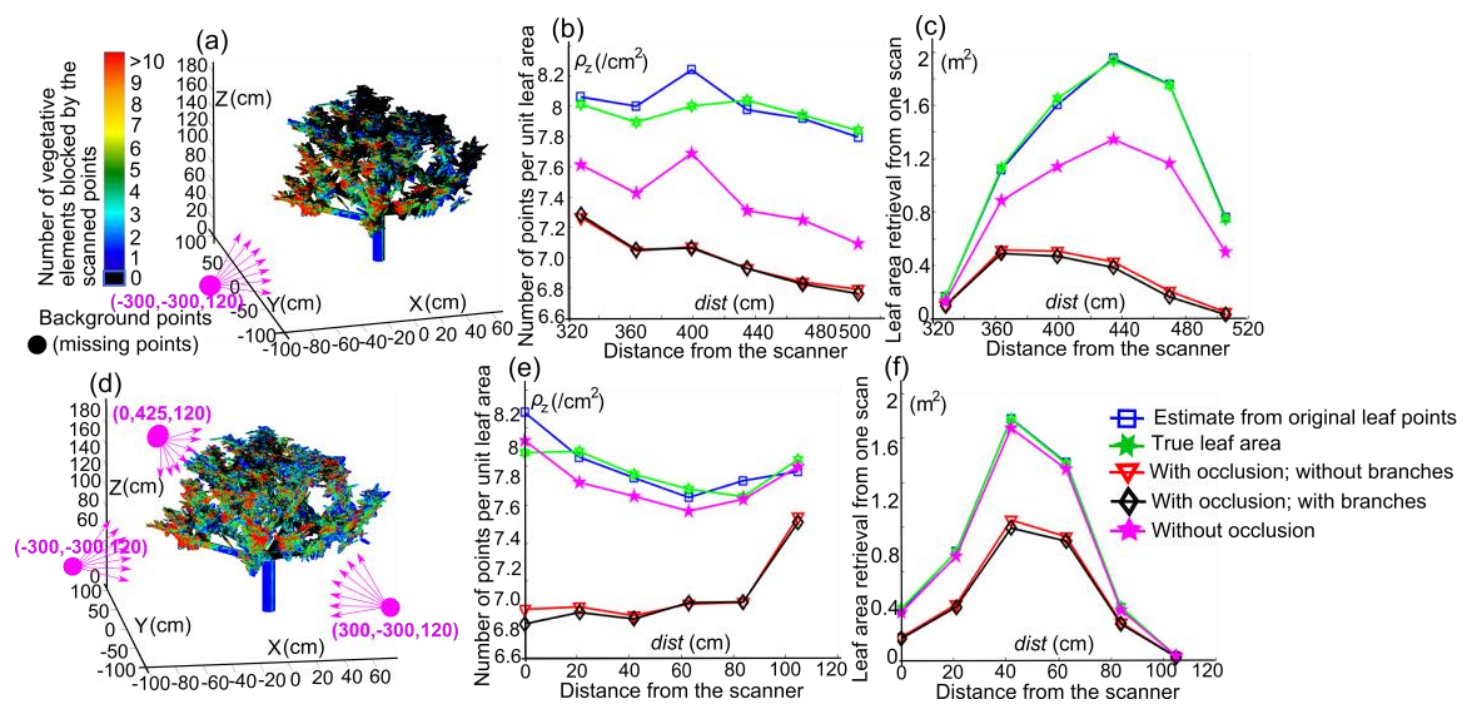

Figure 3. Horizontal scanning profiles representing the ratio $\rho_{z}$ (number of scanned points per $\mathrm{cm}^{2}$ leaf) and retrieved leaf area distribution from the mango tree model. (a) Observed from a perspective view, the mango tree is represented by one virtual scan, where different colours represent the degree to which each scanned point blocks subsequent vegetative elements for each beam, and black represents missing points, which are the occluded points further from the scanner. (b) The calculated ratio $\rho_{z}$ of different segments. (c) The horizontal profiles of leaf area captured by a single scanner. (d) Scanning pattern of three scanners around the target tree. Although from an external viewpoint it appears that comprehensive laser coverage of the tree crown has been obtained, occlusion remains in the centre of the tree crown due to blocking by outer foliage elements. (e) and (f) are equivalent figures to (b) and (c) from three scanning positions represented as distance from the tree crown centre. 


\subsection{Vertical penetration of virtual scans}

The walnut tree is taken as an illustration to reveal the relative degree of occlusion with one versus three TLS positions. Further information on the performance of TLS in occluded structures is predicted by examining estimated leaf area across the vertical profile of the tree crown (Figure 4). The tree crown was divided into six equal-height segments from the lowest to highest detected leaves. $\rho_{z}$ and the total leaf area of each segment were calculated under various scanning patterns. A similar analysis to that seen in the horizontal virtual scans in Figure 3 was conducted but with some important differences. Figures $4 a, b$ and $c$ show the scanning pattern of a single terrestrial scanner alongside the target tree. The majority of laser beams are blocked by the tree crown elements at lower heights. Therefore, occlusion increases with the height of the tree crown, and there is a declining trend of $\rho_{z}$ with increasing distance from a height of 1.2 $m$ (Figure 4b). Following the conversion into leaf area estimates (Figure 4c), a recovery of $60.09 \%$ of total leaf area was achieved for the walnut tree crown without occlusion, but the recovery of TLA with occlusion decreased to $32.50 \%$ or $37.28 \%$ with or without branch occlusion, respectively. The use of a single virtual scan therefore captures only a minority of the leaf area, rendering the accurate estimation of tree crown properties impossible.

The combination of data from three scanning positions around the tree crown reveals similar patterns of $\rho_{z}$ with height (Figure 4e), and the un-scanned vegetative elements exist solely in the centre of the upper tree crown. The point density is highest at lower positions in the tree crown, resulting in a marked improvement in the recovery of true leaf area (Figure 4f). In the absence of occlusion, almost the entire tree crown can be reconstructed and nearly $97.41 \%$ of the tree crown leaf area is retrieved. When occlusion is incorporated, $72.69 \%$ of the tree crown leaf area is detected with and $75.11 \%$ without branch occlusion, which is markedly higher than that from a single scanning point. 

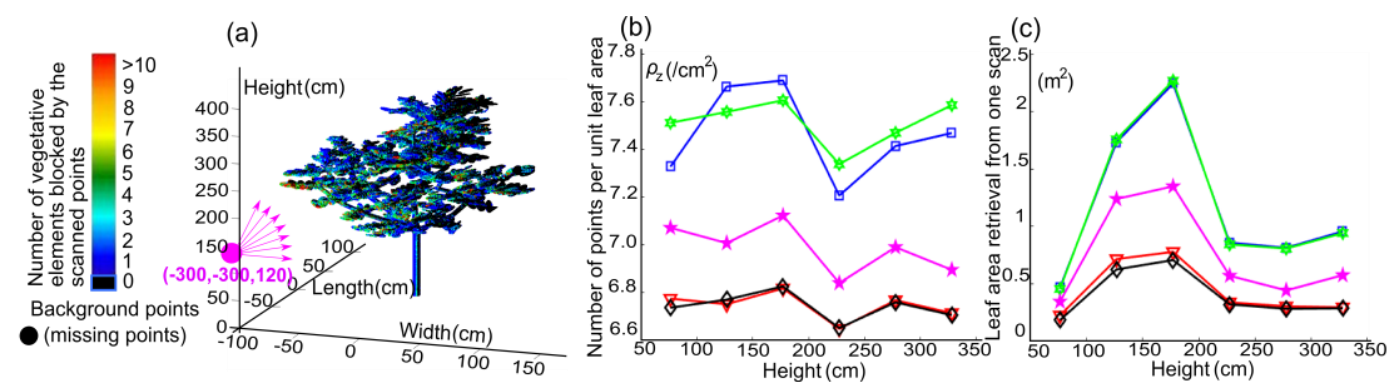

(d)
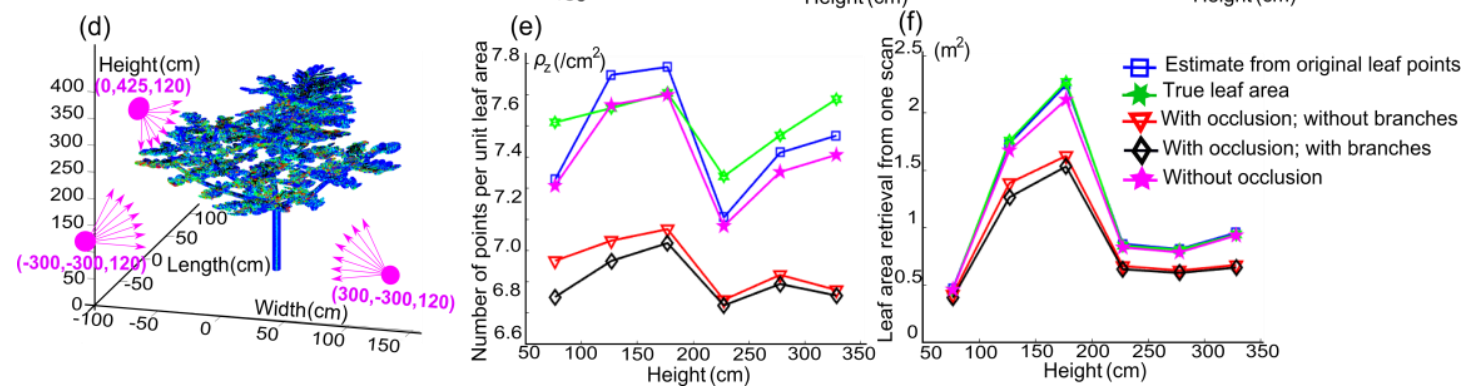

Figure 4. Visualization of the results of the scanning analysis for the walnut tree, which is separated into six layers of equal height with and without occlusion, with and without branches and compared with true leaf area. (a) The tree is scanned from one TLS position, and the degree to which each scanned point blocks vegetative elements located behind the point for each beam is represented by different colours. (b) Ratio $\rho_{z}$ of each height band derived from a single virtual scan decreased with increasing height. (c) Leaf area profiles of each vertical segment from a single virtual scan. (d, e and f) are equivalent figures to $(a, b, c)$ for three TLS positions. 


\subsection{ALS simulation with occlusion}

As shown in the previous sections, the scanning process yields a lower leaf area estimate for the model trees when occlusion is present in the centre and higher parts of the tree crown. This result is due to localized high densities of leaves in the outer and lower parts of the tree crown that are more impenetrable to the laser beams. Thus, a new scanning pattern that included three ground-based virtual scans around a target tree and a virtual scan overhead at a given height was employed to simulate combined TLS and ALS for leaf area retrieval and occlusion quantification. Rubber tree 1 is taken as an example with a scanning pattern similar to that described above but with the inclusion of a newly added aerial virtual scan to predict leaf area captured from both TLS and ALS-based data. The results are shown in Figure 5. An aerial virtual scan is obtained from the overhead position at point $(0,0,5.45 \mathrm{~m})$. Figure 5 a shows that substantial occlusion dominates at lower heights of the tree, and the upper leaves of the tree crown intercept the majority of the laser beams. The light blue dashed line with circular marks in Figures $5 \mathrm{~b}$ and $\mathrm{c}$ represents the $\rho_{z}$ and retrieved leaf area distribution from an aerial virtual scan only. Occlusion decreases at greater heights, and the value of $\rho_{z}$ increases with increasing distance from the ground. The vertical profile of leaf area retrieval from an aerial virtual scan (Figure $5 c$ ) shows that a higher deviation of leaf area estimation occurs at lower heights of the tree crown. In total, $49.28 \%$ of the total leaf area is recovered from an aerial virtual scan, which is larger than the $40.51 \%$ of the leaf area captured from the lateral virtual scan because the tree crown structure allows light to penetrate more deeply into the tree crown from the zenith position. Occlusion is effectively alleviated when the three terrestrial virtual scans and one aerial virtual scan are combined. Thus, occlusion occurs almost entirely inside the tree crown (Figure $5 \mathrm{~d}$ ). Figure 5 e shows a greater $\rho_{z}$ distribution in the upper tree crown (light blue dashed line) than that in the derivation from only three terrestrial virtual scans around the tree crown (black line) because vegetative data retrieval is always deficient from the overhead position when only TLS is adopted. The final leaf area retrieval with comprehensive scanned data coverage (three terrestrial virtual scans and one 
aerial virtual scan added) shown in Figure $5 f$ reaches $90.08 \%$, which is higher than the $73.39 \%$ leaf area retrieval when using only three registered TLS-based virtual scans. The discrepancies in leaf area retrieval between the two scanning patterns become larger with increasing height of the tree because a tall tree decreases the proportion of leaf elements captured from groundbased scanning positions.
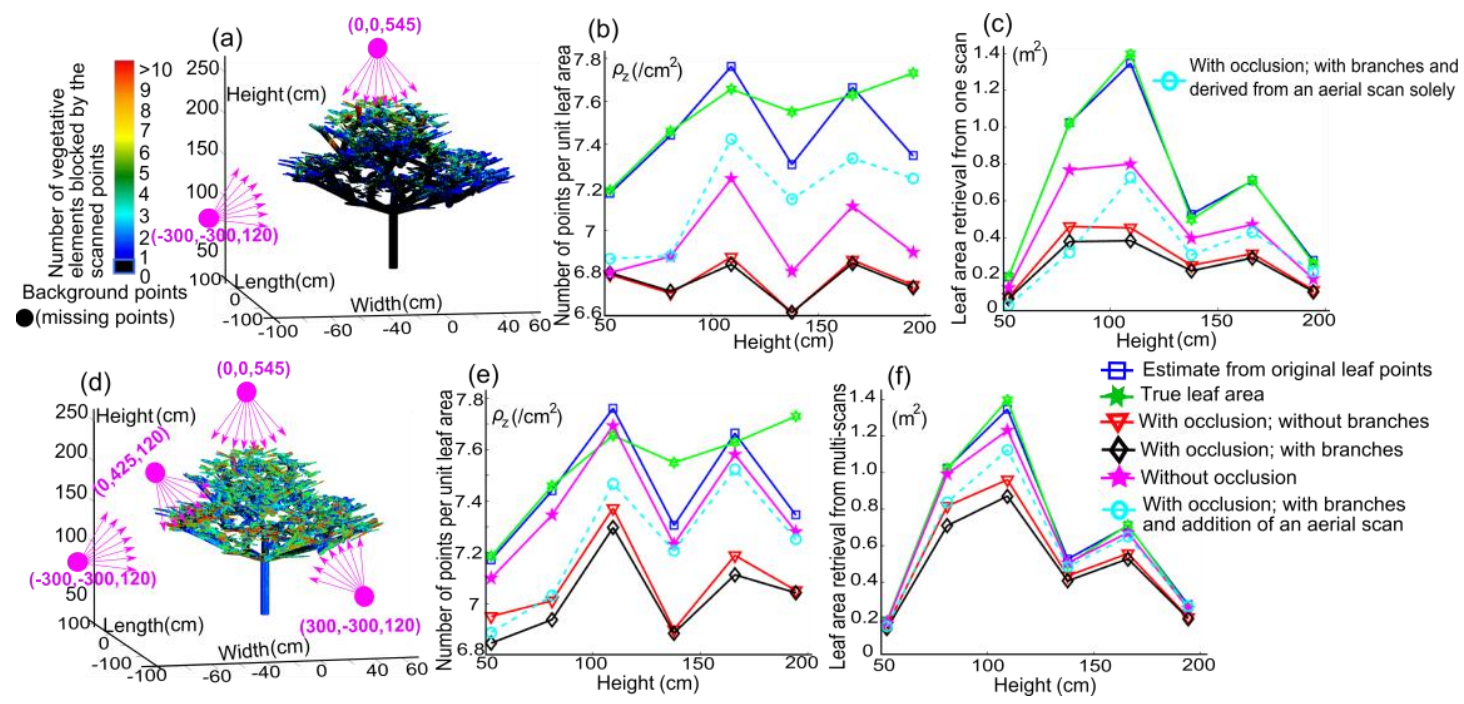

Figure 5. Visualization of scanning results for rubber tree 1 , including the addition of an aerial virtual scan, analysed in the same manner as in Figure 4. (a) Laser beam intercepted by vegetative elements from an aerial scanner. (b) Ratio $\rho_{z}$ of each height band from either a terrestrial or aerial virtual scan. (c) Profiles of leaf area retrieval for each vertical segment from a single terrestrial or aerial virtual scan. (d, e and f) are equivalent figures for the three groundbased virtual scans and with the addition of an aerial virtual scan.

\subsection{Comparison of simulation results for different trees}

The simulations of the five different trees allowed comparisons of the effectiveness of leaf area recovery. The crowns and branches of different trees varied markedly in height and diameter as well as in the number and total area of leaves (Table 1). In Table 2 we present the overall leaf area recovery for each tree based on point clouds assembled from one or three ground-based virtual scans with and without occlusion, with and without branches, and with the 
addition of an aerial virtual scan. The threshold of triangulation for the scanned leaf area calculation is derived from the Supplementary materials section 1.3.

Combining the biological properties of the tree crown shown in Table 1 and our simulation scanning results shown in Table 2, many conclusions were deduced as follows. The mango and walnut tree crowns have nearly the same amount of leaf material, but the mango tree has a lower height and a smaller crown than the walnut tree. As a result, the high leaf area density of the mango tree causes greater occlusion and lower leaf area recovery. Similarly, rubber tree 2 and the apple tree have much larger tree crowns and greater height than the other tree crowns, with an approximately nine-fold increase in the tree crown leaf area and number of leaves relative to any of the other tree crowns. Due to different tree properties and greater distance between the scanner and leaf elements, a finer angular resolution of TLS and ALS was set, and under these conditions the results show a similar degree of self-shading and leaf area retrieval to other tree crowns. For target trees with high angular resolution and close range scanning, with 3 TLS virtual scans around the tree crown, over $60 \%$ of leaf area can be reconstructed from the registered point cloud. This value reaches $72 \%$ for a tree with a lower LAI and smaller crown. Moreover, one overhead virtual scan (ALS pattern) always yields a higher estimation of leaf area for all tree crowns under the influence of occlusion than the leaf area estimation derived from a single TLS-based virtual scan, and the difference between the two patterns ranges from 4.610.6\%. Additionally, the scanned branch elements were less influential on the ALS pattern than in the results from lateral TLS. One explanation for this difference may be that a higher probability of large inclination angles for tree crown leaves optimizes solar radiation absorption, and nonphotosynthetic parts (branches and stems) are not as exposed to available light at smaller zenith angles because they are usually hidden within the tree crown. Furthermore, higher LAI and larger leaf size result in higher occlusion levels. The degree of the overall leaf area retrieval increased to 72.8-90.3\% from three registered TLS-based virtual scans plus one ALS-based virtual scan. When occlusion is not incorporated, almost all leaf elements are captured from three TLS-based virtual scans around the tree crown, which allows for the extrapolation of the total leaf area near the 
true value from these scanned points and verifies that occlusion has a considerable impact during laser scanning data collection.

Table 2. Percentage of the total leaf area recovered when tree crowns were scanned at one or three ground-based scanning positions, with (+) or without (-) branches, with (+) or without (-) occlusion and with or without the supplement of an aerial virtual scan. The final row shows the effectiveness of the triangulation algorithm in reconstructing virtual leaf area from the simulated points.

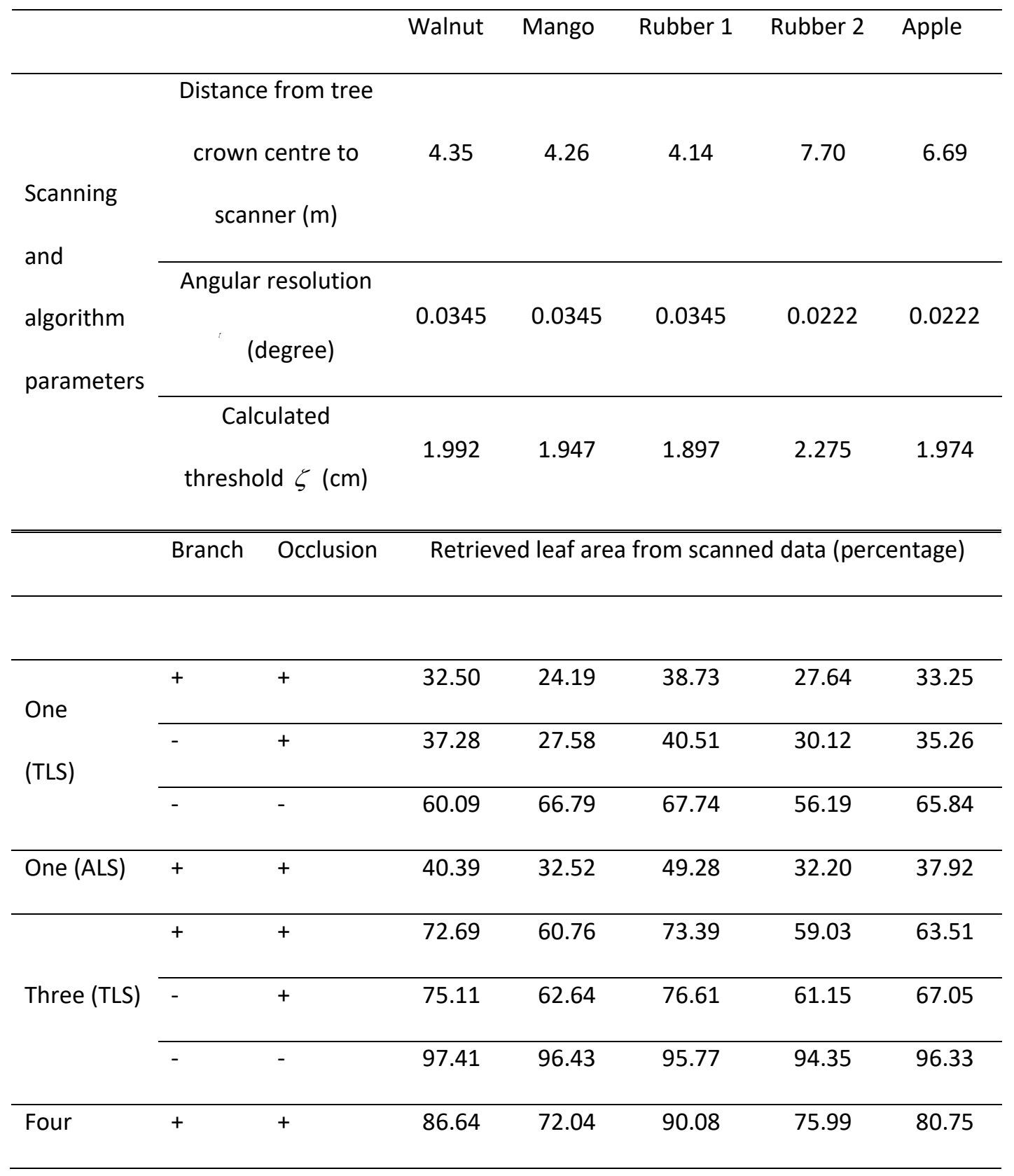


(3TLS+ ALS)

\begin{tabular}{llllll}
\hline Original points & 100.46 & 98.76 & 100.18 & 99.62 & 99.89
\end{tabular}

\section{Discussion}

\subsection{Effect of occlusion on retrieved leaf area}

Leaf area is one of the most important parameters in the biology of trees. Although laser scanning techniques provide detailed information on the three-dimensional structure of trees and forests, the application of laser scanning in the estimation of LAI is complicated by occlusion caused by mutually occluded vegetative elements (Ehbrecht et al., 2016). For instance, the movement of leaves due to wind will cause positional and registration errors in combined point clouds and will increase occlusion (Liang et al., 2016). Trees in forests are also surrounded by other stems and sub-canopy vegetation, which will obstruct terrestrial views. Moreover, airborne laser scanning typically occurs at greater distances and lower point resolutions than in our simulations, and potential occlusion of understory trees is generated by dominant trees and competition between tree crowns from an aerial perspective. As target trees can be scanned from different viewpoints with different point densities caused by beams spreading out in space, methods to infer vegetation leaf area from multiple scans need to account for many factors, including the distance between the target tree and scanners, the parameters of each scan, the occluded foliage elements that exist in the intermediate crown and the discrepancies in the information obtained from different scanning view points. Thus, the existing methods to estimate LAI through voxelization (Béland et al., 2014; Kükenbrink et al., 2017) require further development to quantify the impacts of the occlusion effect.

Many current approaches use the Beer-Lambert law (Woodgate et al., 2016; Zheng and Moskal, 2012) to link laser scanning beam transmittance with foliar surface density to assess LAl. However, accurate estimates of gap fraction, the clumping index of foliage and the validity of the Poisson model in complex structured tree crowns are not easily obtained. Approximations are therefore used instead of empirical values (Olivier and Robert, 2017; Woodgate et al., 2015). The 
difficulty in obtaining reliable reference data also hampers the validation of the approaches developed to assess leaf area and prevents both theoretical and practical issues from being effectively addressed. Direct in situ measurements of LAI are rare due to the complexity, resource requirements and cost involved in the deployment. LAI estimates are also susceptible to bias resulting from the statistical sample size of foliage obtained from tree crowns (Weiss et al., 2004).

Given these constraints, adopting a modelling framework to simulate laser scanning data for realistic vegetation is an attractive option for evaluating the impact of occlusion on leaf area assessments and performing validations. Our modelling framework, which includes vegetation architecture and various scanning patterns, represents a complementary approach for evaluating leaf area and occlusion using reliable reference data.

\subsection{Suggested scanning methods to alleviate occlusion}

The impact of occlusion persists in the laser scanning process to a varying extent between different target trees and scanning patterns. Hence, choosing an optimized scanning strategy to effectively alleviate the occlusion effect is helpful to increase scanned data quality and to improve retrieval of tree leaf area.

The phenotypic characteristics of trees, such as the crown shape, leaf surface properties and gap fraction distribution, are influenced by various factors that include solar radiation, water availability, soil properties, climate type and wind effects on growth. Different tree phenotypes produce different degrees of occlusion under various scanning views. For example, the maximal zenith angle of the sun near the equator places the sun more directly above plants, and for tall trees with flat crowns, more leaves are exposed when the sun is in this position (Duchemin et al., 2018). Hence, aerial scanning patterns above the tree crown can capture more leaf elements than TLS patterns. At high latitudes, the sun is relatively low in the sky, and trees in these regions tend to be cone-shaped, with leaves extending from the top of the tree to the bottom to increase the absorption of sunlight (Duchemin et al., 2018). For these trees, TLS-based scanning 
is a better choice to acquire leaf element information from a lateral view. Our work provides a modelling basis for establishing scanning plans depending on the phenotype of the trees.

Leaf attributes in different positions of tree crowns vary with allometric growth. 'Sun leaves' occur on the irradiated side of the tree crown, and the normal vectors of the leaf surface point almost directly at the sun to ensure direct solar radiation. 'Shade leaves' always exist in tree crowns or on the shady side of the crown where there is limited direct light. Shade leaves have non-uniform normal vectors of the leaf surface to effectively use diffuse solar radiation (de Casas et al., 2011). Hence, a scanner can be placed in a position that faces the shady side of the tree crown and another scanner can be placed facing the side exposed to direct solar radiation. Shade leaves with a non-uniform normal vector of the leaf surface closer to the scanner would allow more laser beams to penetrate the canopy and reach the other (sunny) side, where more leaf surfaces face the direction of the sun, allowing greater exposure of leaf area to the laser beams passing through the crown. In addition, trees can have asymmetric crown structures caused by long-term fixed directions of wind or neighbouring objects that show strong competition for space. In these cases, a TLS facing the side of the tree crown opposing the wind or suppressed by neighbouring objects is preferred due to a larger gap fraction and sparse leaf area density on this side, which optimizes beams passing through the crown and covering more vegetative elements across the tree.

Certain inferences can be deduced from Tables 1 and 2. High scanning resolution and multiangle scan registration can lead to more laser beams entering the tree crown through gaps to optimize the detection of vegetation elements in the crown. Different magnitudes of LAI, tree height and tree crown volume may produce different degrees of mutually occluded vegetative elements and decrease the scanning resolution and laser scanning coverage field relative to the whole tree crown. Based on Table 2, for a small tree (height $<5 \mathrm{~m}$ ) with a larger tree crown $(>3$ $\mathrm{m}^{3}$ ) and smaller LAI $(<3)$, three TLS positions around the tree can capture $80 \%$ of the vegetative elements and less than $20 \%$ compensation is needed for the final leaf area estimation. If the small tree has a larger LAI (>3) and small tree crown, a greater compensation value is necessary 
for leaf area estimation. For tall trees (height $>5 \mathrm{~m}$ ) with a larger tree crown $\left(>10 \mathrm{~m}^{3}\right)$ and lower leaf area $(<3)$, the combination of multi-TLS and ALS is preferred to obtain evenly distributed scanned points of the tree crown covering more than $85 \%$ vegetative elements in the crown. If the LAl of the tall tree is larger than 3 , then more than $25 \%$ of the compensation of the leaf area estimation will likely be needed for scanned data deficiencies in the intermediate tree crown.

\subsection{Application of the method}

The reliability of computer simulation-based methods versus the real scanning process can be determined through a verification trial with a local tree in real world as a reference. Two devices, i.e., an electro-magnetic 3D digitiser Fastrak (Polhemus Inc., Colchester, VT, USA) and a laser scanner (Leica C10 or RiegITM VZ-400), are needed for real tree modelling and benchmark acquisition, respectively. The tree can be modelled using 3D digitizing by Fastrak (Mabrouk and Sinoquet, 1998; Sinoquet et al., 2009), and the virtual tree model can be reconstructed using the corresponding digitized leaf and skeleton information of the real tree, which is taken as the target tree for virtual scanning simulation. For reference data acquisition of TLS and ALS, the scanner can be placed alongside or fixed atop a high place to provide lateral or top-down scanning for the target tree, respectively. The restored virtual scanning scene using our computer simulation, including the relative position of the scanner and the tree, the direction of the scanning view and scanning parameters, is set up according to the real-world arrangement of scanning. Hence, quantitative assessments of results obtained from real and simulation methods (i.e., scanned point number and depth of laser beams penetrating into the tree crown) can be compared to verify the effectiveness of the computer simulation method and provide guidance for further simulation program exploration and upgrades.

The method we propose can be applied to a variety of contexts. It is suitable for most broadleaf trees, but our method has limited value for coniferous trees because needles are difficult to represent using triangle meshes. The scanned targets in our method can be an individual broadleaf tree or expanded into a combination of several trees. In addition, because laser incident 
angles are controllable, different scanning simulations can be conducted with lateral, vertically downward and arbitrarily directional scanning. Our program can be fully extended to a range of scanning contexts. Combined with beam divergence and beam size setting, the time lapse between a laser pulse emission and its return as well as the phase shift between the emitted and received signal can also be incorporated. The simulations can therefore reproduce the performance of various laser sensors, such as Riegl (Xu et al., 2017), Leica (Sun et al., 2016), Velodyne (Atanacio-Jiménez et al., 2011) and Zebedee (Marselis et al., 2016). Our method can simulate a variety of scanning patterns, including UAV-loaded systems (Wallace et al., 2012), mobile terrestrial mapping (Xu et al., 2018) and ALS-TLS cross-scanning (Kükenbrink et al., 2017), and it can also quantify the captured leaf area and occlusion effect for different tree species with various scanning patterns.

Our scanning principle has the potential to be combined with other methods for solving many existing problems: light transmittance modelling among tree crowns with varying structural compositions in terms of leaf area density, leaf distribution and leaf angle distribution; evaluating the impact of various tree crown properties (e.g., clumping index, LAI and gap fraction) on leaf area retrieval using laser scanning techniques; and analysing the ability of intrinsic laser scanner parameters (e.g., angular resolution, beam divergence and return intensity) to characterize specific tree properties.

\section{Conclusion}

Using a dataset of typical trees for which the positions, sizes and directions of all leaves and branches were known we were able to construct real tree models and provide validation data to evaluate the efficacy of laser scanning techniques in estimating the total leaf area of individual trees. Designing an optimized ray intersection algorithm with adjustable parameters to simulate the laser scanning process and to obtain point clouds using various scanning patterns enables detailed data acquisition. The leaf area covered by laser beams is calculated synchronously from 
the scanned points using a 3D triangulation method with automatic adaptive threshold selection to provide accurate evaluations of the degree of occlusion using various scanning patterns.

The results showed that only $25-38 \%$ of leaf area was retrieved and occlusion occurred on leaves distal to the scanner when the target tree was scanned from a single terrestrial position. When three terrestrial virtual scans were performed around a tree, the accuracy of leaf area recovery reached approximately $60-72 \%$, and occlusion was restricted to the crown centre. If a supplementary aerial virtual scan was included, leaf area recovery increased to $72-90 \%$, depending on the leaf area index, tree crown volume and leaf area density. Our approach shows promise for tree structural measurement using laser scanning techniques in practice at the scale of individual tree leaves. With appropriate parameterization, the approach can be applied to any broad-leaf tree species and any scanning patterns and can be extended to both single- and multiple-return devices. With the development of computer graphics algorithms, our approach therefore holds potential for accurate field measurements of tree leaf area.

Acknowledgements: The authors would like to thank the National Natural Science Foundation of China (31770591, 41701510), the National Key R\&D program of China (2017YFD0600904), and the China Postdoctoral Science Foundation (2016 M601823). In addition, we are grateful for the experimental devices provided by the Priority Academic Program Development of Jiangsu Higher Education Institutions.

Author Contributions: Ting Yun and Lianfeng Xue wrote the programs and developed the methods; Feng An and Weizheng Li analysed the results and accuracy assessment; Bangqian Chen and Lin Cao participated in the coordination of the study and edited the manuscript; SP provided field data; MJS and MPE designed and supervised the original study; all authors contributed to the drafts of the manuscript and approved the final version for publication.

\section{References}


Alonzo, M., Bookhagen, B., McFadden, J.P., Sun, A., Roberts, D.A., 2015. Mapping urban forest leaf area index with airborne lidar using penetration metrics and allometry. Remote Sens. Environ. 162, 141-153. https://doi.org/10.1016/j.rse.2015.02.025

Asvadi, A., Premebida, C., Peixoto, P., Nunes, U., 2016. 3D Lidar-based static and moving obstacle detection in driving environments: An approach based on voxels and multi-region ground planes. Rob. Auton. Syst. 83, 299-311.

Atanacio-Jiménez, G., González-Barbosa, J.-J., Hurtado-Ramos, J.B., Ornelas-Rodríguez, F.J., JiménezHernández, H., García-Ramirez, T., González-Barbosa, R., 2011. Lidar velodyne hdl-64e calibration using pattern planes. Int. J. Adv. Robot. Syst. 8, 59.

Bailey, B.N., Mahaffee, W.F., 2017. Rapid measurement of the three-dimensional distribution of leaf orientation and the leaf angle probability density function using terrestrial LiDAR scanning. Remote Sens. Environ. 194, 63-76.

Béland, M., Baldocchi, D.D., Widlowski, J.L., Fournier, R. a., Verstraete, M.M., 2014. On seeing the wood from the leaves and the role of voxel size in determining leaf area distribution of forests with terrestrial LiDAR. Agric. For. Meteorol. 184, 82-97. https://doi.org/10.1016/j.agrformet.2013.09.005

Boegh, E., Soegaard, H., Broge, N., Hasager, C.B., Jensen, N.O., Schelde, K., Thomsen, A., 2002. Airborne multispectral data for quantifying leaf area index, nitrogen concentration, and photosynthetic efficiency in agriculture. Remote Sens. Environ. 81, 179-193.

Chaivivatrakul, S., Tang, L., Dailey, M.N., Nakarmi, A.D., 2014. Automatic morphological trait characterization for corn plants via 3D holographic reconstruction. Comput. Electron. Agric. 109, 109-123. https://doi.org/10.1016/j.compag.2014.09.005

Chen, J.M., Chen, X., Ju, W., Geng, X., 2005. Distributed hydrological model for mapping evapotranspiration using remote sensing inputs. J. Hydrol. 305, 15-39.

Côté, J.F., Fournier, R. a., Frazer, G.W., Olaf Niemann, K., 2012. A fine-scale architectural model of trees to enhance LiDAR-derived measurements of forest canopy structure. Agric. For. Meteorol. 166-167, 72-85. https://doi.org/10.1016/j.agrformet.2012.06.007

de Casas, R.R., Vargas, P., Pérez-Corona, E., Manrique, E., García-Verdugo, C., Balaguer, L., 2011. Sun and shade leaves of Olea europaea respond differently to plant size, light availability and genetic variation. Funct. Ecol. 25, 802-812.

Duchemin, L., Eloy, C., Badel, E., Moulia, B., 2018. Tree crowns grow into self-similar shapes controlled by gravity and light sensing. J. R. Soc. Interface 15, 20170976.

Ehbrecht, M., Schall, P., Juchheim, J., Ammer, C., Seidel, D., 2016. Effective number of layers: A new measure for quantifying three-dimensional stand structure based on sampling with terrestrial LiDAR. For. Ecol. Manage. 380, 212-223.

Kükenbrink, D., Schneider, F.D., Leiterer, R., Schaepman, M.E., Morsdorf, F., 2017. Quantification of hidden canopy volume of airborne laser scanning data using a voxel traversal algorithm. Remote Sens. Environ. 194, 424-436.

Lati, R.N., Filin, S., Eizenberg, H., 2013. Plant growth parameter estimation from sparse 3D reconstruction based on highly-textured feature points. Precis. Agric. 14, 586-605. https://doi.org/10.1007/s11119-013-9317-6

Li, Y., Guo, Q., Su, Y., Tao, S., Zhao, K., Xu, G., 2017. Retrieving the gap fraction, element clumping index, and leaf area index of individual trees using single-scan data from a terrestrial laser scanner. ISPRS J. Photogramm. Remote Sens. 130, 308-316.

Liang, X., Kankare, V., Hyyppä, J., Wang, Y., Kukko, A., Haggrén, H., Yu, X., Kaartinen, H., Jaakkola, A., Guan, F., 2016. Terrestrial laser scanning in forest inventories. ISPRS J. Photogramm. Remote Sens. 115, 63-77.

Liu, J., Skidmore, A.K., Heurich, M., Wang, T., 2017. Significant effect of topographic normalization of airborne LiDAR data on the retrieval of plant area index profile in mountainous forests. ISPRS J. Photogramm. Remote Sens. 132, 77-87.

Lu, X., Guo, Q., Li, W., Flanagan, J., 2014. A bottom-up approach to segment individual deciduous trees using leaf-off lidar point cloud data. ISPRS J. Photogramm. Remote Sens. 94, 1-12. https://doi.org/10.1016/j.isprsjprs.2014.03.014

Ma, L., Zheng, G., Eitel, J.U.H., Magney, T.S., Moskal, L.M., 2017. Retrieving forest canopy extinction coefficient from terrestrial and airborne lidar. Agric. For. Meteorol. 236, 1-21. 
Mabrouk, H., Sinoquet, H., 1998. Indices of light microclimate and canopy structure of grapevines determined by $3 \mathrm{D}$ digitising and image analysis, and their relationship to grape quality. Aust. J. Grape Wine Res. 4, 2-13.

Marselis, S.M., Yebra, M., Jovanovic, T., van Dijk, A.I.J.M., 2016. Deriving comprehensive forest structure information from mobile laser scanning observations using automated point cloud classification. Environ. Model. Softw. 82, 142-151.

Méndez, V., Catalán, H., Rosell-Polo, J.R., Arnó, J., Sanz, R., 2013. LiDAR simulation in modelled orchards to optimise the use of terrestrial laser scanners and derived vegetative measures. Biosyst. Eng. 115, 7-79. https://doi.org/10.1016/j.biosystemseng.2013.02.003

Nilson, T., 1971. A theoretical analysis of the frequency of gaps in plant stands. Agric. Meteorol. 8, 2538.

Olivier, M.-D., Robert, S., 2017. A method to quantify canopy changes using multi-temporal terrestrial lidar data: Tree response to surrounding gaps. Agric. For. Meteorol. 237, 184-195.

Pesci, A., Teza, G., Bonali, E., 2011. Terrestrial laser scanner resolution: Numerical simulations and experiments on spatial sampling optimization. Remote Sens. 3, 167-184. https://doi.org/10.3390/rs3010167

Sanz, R., Llorens, J., Arnó, J., Planas, S., Román, C., Rosell-Polo, J.R., 2018. LIDAR and non-LIDAR-based canopy parameters to estimate the leaf area in fruit trees and vineyard. Agric. For. Meteorol. 260, 229-239.

Sinoquet, H., Pincebourde, S., Adam, B., Donès, N., Phattaralerphong, J., Combes, D., Ploquin, S., Sangsing, K., Kasemsap, P., Thanisawanyangkura, S., 2009. 3-D maps of tree canopy geometries at leaf scale. Ecology 90, 283.

Sun, Y., Liang, X., Liang, Z., Welham, C., Li, W., 2016. Deriving merchantable volume in poplar through a localized tapering function from non-destructive terrestrial laser scanning. Forests 7, 87.

Van der Zande, D., Stuckens, J., Verstraeten, W.W., Mereu, S., Muys, B., Coppin, P., 2011. 3D modeling of light interception in heterogeneous forest canopies using ground-based LiDAR data. Int. J. Appl. Earth Obs. Geoinf. 13, 792-800. https://doi.org/10.1016/j.jag.2011.05.005

Van Leeuwen, M., Coops, N.C., Hilker, T., Wulder, M.A., Newnham, G.J., Culvenor, D.S., 2013. Automated reconstruction of tree and canopy structure for modeling the internal canopy radiation regime. Remote Sens. Environ. 136, 286-300.

Vauhkonen, J., Holopainen, M., Kankare, V., Vastaranta, M., Viitala, R., 2016. Geometrically explicit description of forest canopy based on 3D triangulations of airborne laser scanning data. Remote Sens. Environ. 173, 248-257.

Wallace, L., Lucieer, A., Watson, C., Turner, D., 2012. Development of a UAV-LiDAR system with application to forest inventory. Remote Sens. 4, 1519-1543.

Watt, A.H., Watt, A., 2000. 3D computer graphics. Addison-Wesley Reading.

Weiss, M., Baret, F., Smith, G.J., Jonckheere, I., Coppin, P., 2004. Review of methods for in situ leaf area index (LAI) determination: Part II. Estimation of LAI, errors and sampling. Agric. For. Meteorol. 121, 37-53.

Woodgate, W., Armston, J.D., Disney, M., Jones, S.D., Suarez, L., Hill, M.J., Wilkes, P., Soto-Berelov, M., 2016. Quantifying the impact of woody material on leaf area index estimation from hemispherical photography using 3D canopy simulations. Agric. For. Meteorol. 226, 1-12.

Woodgate, W., Disney, M., Armston, J.D., Jones, S.D., Suarez, L., Hill, M.J., Wilkes, P., Soto-Berelov, M., Haywood, A., Mellor, A., 2015. An improved theoretical model of canopy gap probability for Leaf Area Index estimation in woody ecosystems. For. Ecol. Manage. 358, 303-320.

Xu, S., Wang, R., Zheng, H., 2017. Road Curb Extraction From Mobile LiDAR Point Clouds. IEEE Trans. Geosci. Remote Sens. 55, 996-1009.

Xu, S., Xu, S., Ye, N., Zhu, F., 2018. Individual stem detection in residential environments with MLS data. Remote Sens. Lett. 9, 51-60.

Yun, T., An, F., Li, W., Sun, Y., Cao, L., Xue, L., 2016. A novel approach for retrieving tree leaf area from ground-based LiDAR. Remote Sens. 8. https://doi.org/10.3390/rs8110942

Zhao, K., García, M., Liu, S., Guo, Q., Chen, G., Zhang, X., Zhou, Y., Meng, X., 2015. Terrestrial lidar remote sensing of forests: Maximum likelihood estimates of canopy profile, leaf area index, and leaf angle distribution. Agric. For. Meteorol. 209-210, 100-113.

https://doi.org/10.1016/j.agrformet.2015.03.008 
Zheng, G., Moskal, L.M., 2012. Computational-geometry-based retrieval of effective leaf area index using terrestrial laser scanning. IEEE Trans. Geosci. Remote Sens. 50, 3958-3969.

https://doi.org/10.1109/TGRS.2012.2187907 


\section{Supporting Information: Appendix S1}

for 'Simulation of multi-platform LiDAR for assessing total leaf area in tree crowns' Yun et al. 2019. Agricultural and Forest Meteorology

\section{Supplementary Materials}

\subsection{Rapid simulations of various laser scanning patterns}

To generate a point cloud from the modelled tree crowns, virtual laser scans were conducted via simulation. Each virtual scan was parameterized according to the specifications of a Leica scanning station C10 (Leica Geosystems AG, Heerbrugg, Switzerland) with a horizontal angle of $\alpha \in\left\{0^{\circ}, 360^{\circ}\right\}$, a vertical angle of $\beta \in\left\{0^{\circ}, 180^{\circ}\right\}$, and a minimal angle increment (horizontal and vertical) of $\tau \in\left\{0.014^{\circ}, 0.229^{\circ}\right\}$. As this was a computer simulation, the positional accuracy and effective range were not influenced by signal attenuation or any external environmental interference. The maximum laser range can be set to any value. Three scanners performing TLS were placed in sequence around the target tree, and the base of each virtual tree was placed at position $\{x=0, y=0\}$. An additional ALS position was subsequently added with the same scanning parameters. The distance between each scanner and the centre of the target tree crown was set to the same value.

The computation steps of the laser scanning simulation were as follows. The virtual scanner at position $S$ projects a beam $R$ with an angle $\alpha$ on the $X, Y$ plane (azimuth) and $\beta$ on the $X, Z$ plane (vertical angle), with the directional vector $\vec{D}=\{1, \tan \alpha, \tan \beta\}$. Then, the pointslope form of each laser beam can be expressed as $\left(x-S_{x}\right) / 1=\left(y-S_{y}\right) / \tan \alpha=\left(x-S_{z}\right) / \tan \beta=t$. The intersection of rays with basic geometric primitives (leaf surfaces and branch cylinders) results in a heavy computational burden. In section 2.2 , the leaf surface and branch elements were transformed into sampling points. Thus, in our method, the laser scanning simulation consisted in finding the intersection between each beam and point set $P_{t}$, which greatly reduced the computational complexity and improved the efficiency of our scanning algorithm.

By altering angles $\alpha$ and $\beta$ with a minimal angle increment $\tau$, a point cloud representing interaction points between beams and the virtual tree is created. Since occlusion occurs when 
beams strike a target, only the nearest intersection point on each beam to the scanner $S$ was identified as the scanned point. All subsequent intersections were considered as occluded.

Fig. S1 shows an example of our scanning process for the mango tree model. First, a single scanner is placed alongside the virtual tree in the simulation (Fig. S1 a and b) to illustrate occlusion in both the vertical and horizontal dimensions. The scanner is placed at approximately $1.2 \mathrm{~m}$ height and a distance of approximately $4 \mathrm{~m}$ from the small tree crown centre (walnut tree, mango tree and rubber tree1) and approximately $7 \mathrm{~m}$ away from the larger tree crown centre (rubber tree2 and apple tree). This is a reasonable setting for TLS in reality. The scanning angular resolution $\tau$ is set to 0.0345 degrees for smaller tree crowns and 0.0222 degrees for larger tree crowns, which is similar to the set parameter of a fine resolution scan using Leica C10. The spatial resolution of scanned points is inversely proportional to the acquisition distance as laser beams spread out in space. Hence, the model trees were separated into different layers to approximate the spatial resolutions of the scanned points within each layer according to different scanning patterns. For one scan from a lateral perspective, the trees were divided into six annular sectors of even width through the tree crown from the points nearest and farthest from the scanner (Fig. S1 b). Next, the simulation was repeated with three evenly spaced scanners surrounding the tree (Fig. S1 c and d), after which all virtual scans were combined into a single coordinate system through registration. In this case, model trees were divided into six ring segments based on the distance of points from the tree crown centre. To determine the vertical patterns of attenuation, tree crowns were divided into six equal-height segments from the lowest to the highest detected leaves (Fig. S1 e and f). 

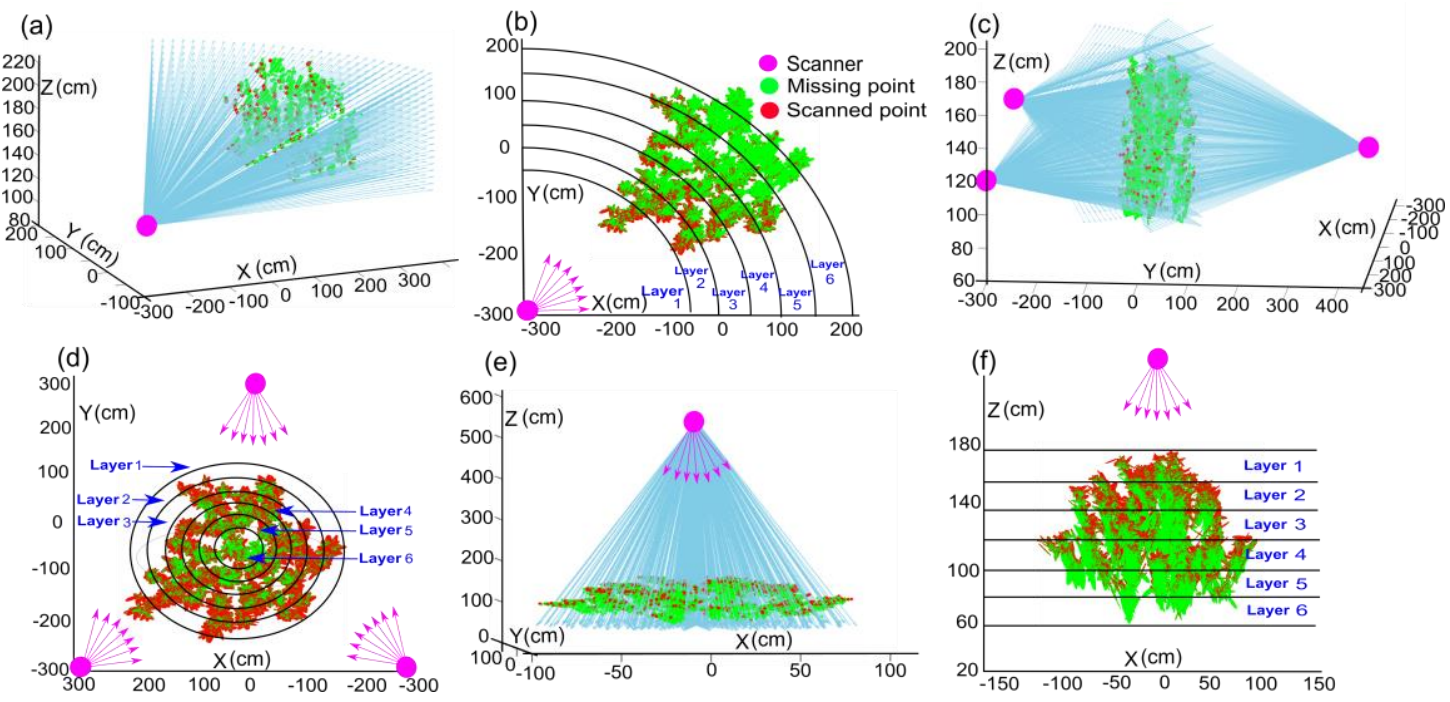

Fig. S1. Schematic diagrams illustrating the laser scanning simulation for a model tree (mango tree) and the division of point clouds for analysis. (a) Single virtual scan showing the intersection of beams with the partial sampling points close to the scanner; (b) horizontal slicing of the tree crown to analyse the efficacy of one virtual scan for leaf area retrieval; (c) scanning from three positions around the target tree showing occlusion results for missing points in the intermediate tree crown; (d) division of the tree crown into six layers according to the distance from the scanner to the tree crown centre for occlusion analysis; (e) the scanned points are mainly distributed in the upper parts of the tree crown from a top-down airborne scanning set-up; (f) division of the tree crown into six layers of equal heights from the top of the crown to the ground for occlusion assessment.

\subsection{Beam divergence definition}

When laser pulses emitted by a laser scanning instrument are used for light transmission through a medium, the size of the pulse's cross section should be considered since partial intersection of pulses leads to a greater number of scanned points. In our scanning simulation, the laser beam was set to $7 \mathrm{~mm}$ in diameter as it left the scanner, with a beam divergence of 0.5 mrad, which gives a $10 \mathrm{~mm}$ beam width at a $10 \mathrm{~m}$ range. When a beam hits the edge of a surface, part of the beam is backscattered towards the scanner, and the residual signal will travel along the path until intercepted by other vegetative elements. When the second hit of a beam occurs 
on an edge point of the leaf surface, a second echo is triggered and the partial beam continues with its remaining energy. This strategy can be employed to manage multi-echo TLS information. The maximum number of triggering returns per beam was set to three due to the attenuation of beam energy after each splitting event.

Fig. S2 shows the proportions of first, second and last returns in one virtual scan obtained from TLS and ALS. The edge area of the leaf surface per unit area increases with decreased leaf size and increased LAI. The quantitative comparison of scanning results in Fig. S2 shows that the scanned trees with smaller average leaf size (walnut tree and rubber tree 1) and higher values of LAI (mango tree) have a higher proportion of scanned points obtained from partial hits. A larger crown of the target tree (rubber tree 2 and apple tree) also improves the probability of multiecho occurrence. In addition, the proportion of multi-echo (second and last return) from ALS is usually smaller than the magnitude from TLS. The reason for this effect is that plant leaf surfaces face the sun to optimize photosynthesis, causing most of the leaf surface to be oriented towards the zenith to increase the number of first-return points, which leads to a reduction in the proportion of points belonging to the second- and third-return categories.
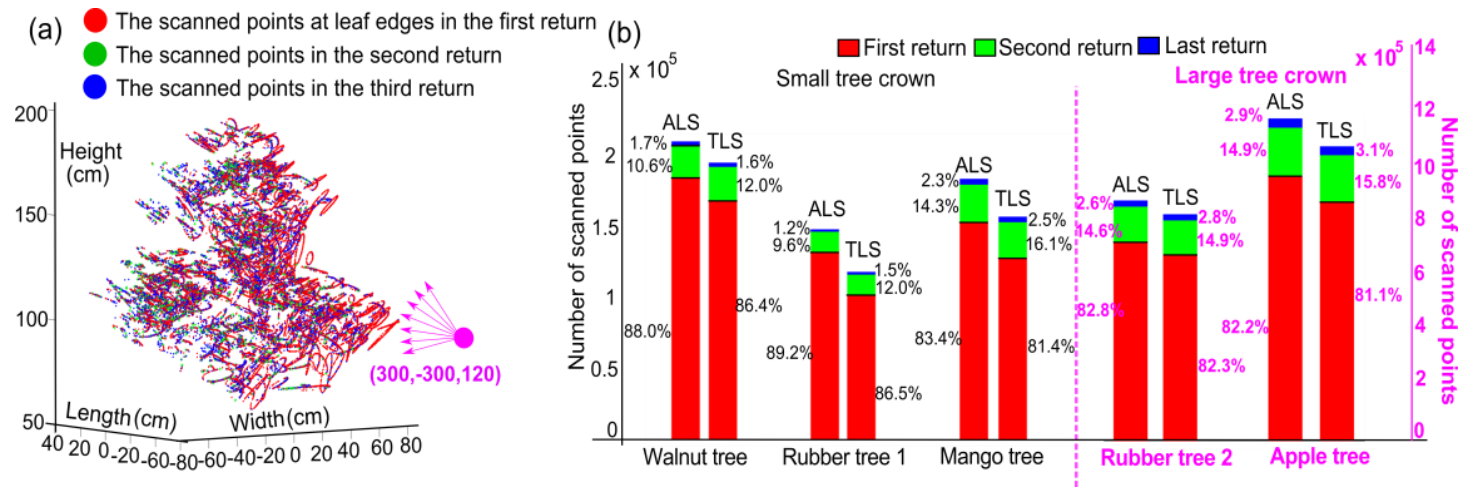

Fig. S2. Illustration showing the multi-echo scanning results of our program and experimental comparison for different tree crowns with various attributes. (a) The scanned points for a sample tree (rubber tree 1), where the red points represent the 'partial hits' of laser beams in the edge area of leaves and the green and blue points represent the scanned points from a multi-echo system. (b) Stacked bar chart with dual-Y axes shows the number of scanned points within each return using TLS or ALS patterns. The numbers alongside each bin represent the percentage of 
scanned points from each return. The left $\mathrm{Y}$-axis marks the number of scanned points in each return for small tree crowns (walnut tree, rubber tree 1 and mango tree, with their total crown leaf area $<10 \mathrm{~m}^{2}$, see Table 1), and the right $\mathrm{Y}$-axis marks the equivalent values for larger tree crowns (rubber tree 2 and apple tree, with their crown leaf area $>35 \mathrm{~m}^{2}$, see Table 1). The bars in each column with different colours represent the proportion of scanned points in each return for the different target trees.

\subsection{Triangulation with adaptive threshold selection}

To deduce the total leaf area within the tree crown, an estimation of the point density of scanned points per unit leaf area obtained from different scanning patterns is required. Hence, a ratio $\rho_{z}$ of the number of points to foliage area within each layer was proposed using Delaunay triangulation to assess the density of scanned points.

For the assessment of $\rho_{z}$, the leaf surfaces were reconstructed from incomplete and occluded point clouds via Delaunay triangulation. Usually the high-density scanned points lie on the leaf surfaces, forming triangles with small perimeters. In the gaps or occluded area, no points exist, but the triangles are also formed by the scanned points around the area and have larger perimeters. Therefore, the perimeter length of each triangle was calculated and compared with a threshold $\zeta$. If the perimeter $L_{A}>\zeta$, then triangle $A$ was considered to belong to a gap or occluded area needed to be discarded. If the perimeter $L_{A} \leq \zeta$, then triangle $A$ was taken as a section of the scanned leaf area.

An original approach was proposed to adaptively assign an appropriate value for the threshold $\zeta . \zeta$ is correlated with the scanned point spacing on every leaf plane, which is affected by three parameters, i.e., the scanning angular resolution $\tau$, the distance dist from the scanner to each leaf and the angles $\varphi$ between the normal vector of the hit leaf surface and the direction of the incident laser beams (Fig. S3 a and b). For all plants in nature, the orientation of each leaf, including the inclination and azimuth angles, and the incident angles of laser beams are assumed to be distributed randomly, resulting in an uncertain value of $\varphi$ for each leaf, and 
dist is also not fixed. Hence, a computer simulation technique was adopted to analyse the relationship between the threshold $\zeta$ and other parameters such as $\varphi$, dist and $\tau$. An experiment includes a virtual scanner at a located position emitting numerous laser beams with distance dist to the scanned leaf, scanning angular resolution $\tau$ and the orientation of the scanned leaf surface changed to examine the variation in spot spacing of scanned points on the leaf surface (Fig. S3 a and b).

The minimum spot spacing occurs when the leaf surface is perpendicular to the incident laser beams (green surface in Fig. $\mathrm{S} 3 \mathrm{a}$ and $\mathrm{b}$ ), and the perimeters of any right triangles $A$ (blue) and $B$ (red) with vertices of scanned points $p_{i, j}, p_{i, j+1}, p_{i+1, j}$ and $p_{i, j}, p_{i+1, j}, p_{i+1, j+1}$, respectively, on the leaf surface can be deduced from computer graphics (Watt and Watt, 2000). After the change in dist, $\tau$ and $\varphi$ (induced by spatial rotation of the leaf surface), any triangle $A\left(p_{i, j}, p_{i, j+1}, p_{i+1, j}\right) \quad$ or $B\left(p_{i, j}, p_{i+1, j}, p_{i+1, j+1}\right) \quad$ converted into $A^{r}\left(p_{i, j}^{r}, p_{i, j+1}^{r}, p_{i+1, j}^{r}\right) \quad$ or $B^{r}\left(p_{i, j}^{r}, p_{i+1, j}^{r}, p_{i+1, j+1}^{r}\right)$, respectively, with the perimeters of the triangles $L$ changed accordingly. The change $\mu$ in the triangle perimeters versus the variation in different parameters dist, $\tau$ and $\varphi$ can be deduced using computer graphics methods, which is shown in Fig. S3 c, d and e. $k$ in equation (1) represents the $k$ th triangle in the total $m$ number of triangles covering the whole leaf surface.

$$
\mu=\sum_{k=1}^{m} L_{A_{k}^{r}} / \sum_{k=1}^{m} L_{A_{k}}
$$

Fig. S3 c shows that the value of $\mu$ remains relatively stable as $\varphi$ varies from 0 to $70^{\circ}$ (light blue area) and increases sharply as $\varphi \geq 70^{\circ}$. When $\varphi \geq 70^{\circ}$, the scanned leaf surface is nearly parallel to the direction of the incident laser beam and the projected area of the leaf surface along the direction of the incident laser beam is narrow, resulting in a very sparse density of the scanned point covering the leaf surface and, hence, difficulty in leaf area retrieval. Therefore, $\varphi=70^{\circ}$ was selected as the evaluation boundary for quantifying the value $\mu$. As shown in Fig. S3 d, the value of $\mu$ remains steady as the angular resolution of $\tau$ varies from 0.01 
to 0.25 degrees (marked in the blue area); this range is reasonable for all scanners and scanning processes and has general applicability in the use of scanning instruments. Fig. S3 e shows that $\mu$ is independent of dist. Moreover, a positive correlation between the side length $a_{1}$ of the triangle $A$ or $B$ and $\tau$ or dist is shown in Fig. S3 $\mathrm{d}$ and e, respectively. As a consequence, the average value of $\mu$ is calculated as nearly 2.23 from equation (1) when $\tau$ ranges from 0.001 to 0.25 degrees and $\varphi=70^{\circ}$. Then, a threshold $\zeta$ to determine whether a triangle belongs to the leaf surface, gap or occlusion area is deduced from equation (2).

$$
\zeta=(\mu+\varepsilon) \cdot\left(L_{A}+L_{B}\right) / 2=(2.23+\varepsilon) \cdot(2+\sqrt{2}) a_{1}+\varepsilon=7.61 \cdot a_{1}+\varepsilon \cdot(2+\sqrt{2}) a_{1}
$$

where $L_{A}=L_{B}=(2+\sqrt{2}) a_{1}$ and $a_{1}$ is the length of $\left|p_{i, j}, p_{i, j+1}\right|$ or $\left|p_{i, j}, p_{i+1, j}\right|$. The value of $a_{1}$ is related to $\tau$ and dist and can be deduced from the Law of Sines $a_{1}=\sin (\tau) \cdot \operatorname{dist} / \sin ((\pi-\tau) / 2) \cdot \varepsilon \in[-0.4,0.4]$ is the correction coefficient, which is related to the leaf size, gap fraction and clumping index of leaf elements of the target tree crowns. If the tree has a smaller leaf size and higher leaf clumping index, $\varepsilon$ is assigned a smaller value to lower the value of threshold $\zeta$, which prevents the gaps between leaves from being incorrectly considered as part of the leaf surface. If the tree has larger leaves and a smaller leaf clumping index, $\varepsilon$ is assigned a larger value to accurately depict each leaf area. 

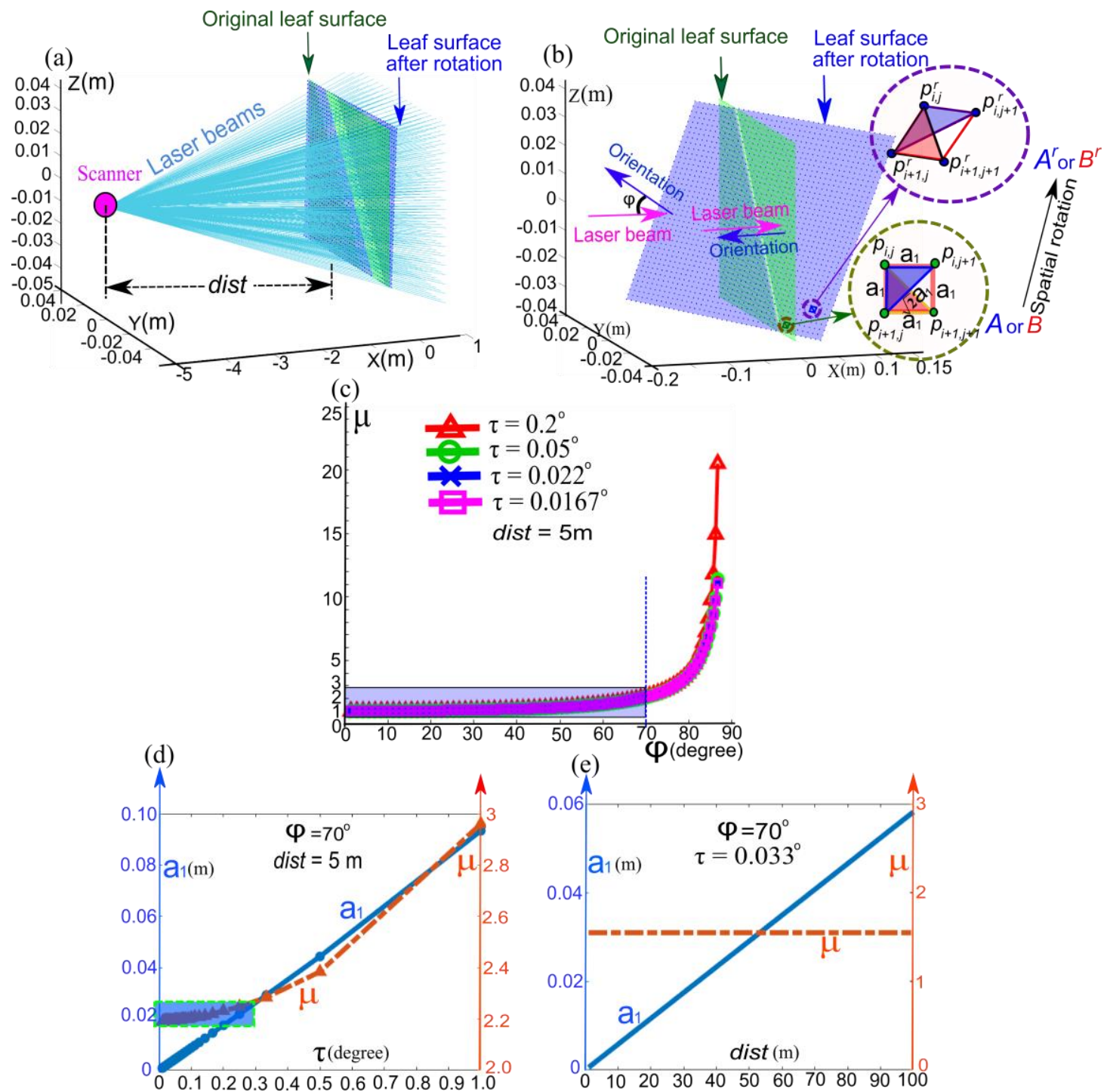

Fig. S3. Schematic representation illustrating the relationship between spot spacing of the scanned points on each leaf surface and other scanning-related parameters. (a) The laser beams are emitted from a scanner at a given position with scanning angular resolution $\tau$. The target leaf plane, with dist metres away from the scanner, changes its orientation to show the variation in spot spacing using the line-plane intersection algorithm. (b) The original (perpendicular to the incident laser beams) and rotated leaf planes with the scanned points on the planes and triangulation at higher resolution. The change in the perimeter of each triangle determines the threshold $\zeta$ in leaf area estimation. (c) The relationship between $\mu$ and the angle $\varphi$ between the beam and leaf surface. Under varying angular resolution $\tau$, the value of $\mu$ remains relatively stable when $\varphi \leq 70^{\circ}$. (d) The dual-y-axis plot shows variations in $a_{1}$ and $\mu$ versus $\tau$, 
and the value of $\mu$ varies little when $\tau$ varies from 0.01 to 0.25 degrees (blue zone). (e) The dual-y-axis plot shows that $\mu$ is independent of dist while $a_{1}$ is positively correlated with dist.

\subsection{Conversion of scanned points to leaf surfaces}

Fig. S4 illustrates our triangulation process. Individual leaves are composed of triangles with vertices formed by scanned points (Fig. S4 a). Filtering within tree crowns by the threshold not only recognizes the blind region caused by perspective occlusion, which would inflate leaf area estimates, but also removes the gaps between leaves (Fig. S4 b). This procedure produces a final set of triangles from which the leaf surface area covered by laser beams can be estimated (Fig. S4 c). Therefore, the ratio $\rho_{z}$ between the number of points and the corresponding leaf area covered by the scanned points within each layer $z$ was calculated. The total leaf area TLA for all scanned points was then estimated from the number of scanned points recorded $N_{z}$ within each layer and divided by the ratio $\rho_{z}$ in each layer. The equation is listed below, where $n$ represents the number of partition layers of the tree crown, and in our program, $n=6$.

$$
T L A=\sum_{z=1}^{n} \frac{N_{z}}{\rho_{z}}
$$

(a)

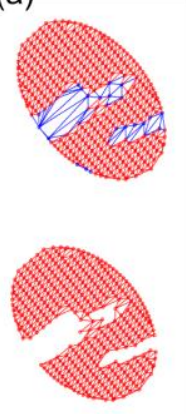

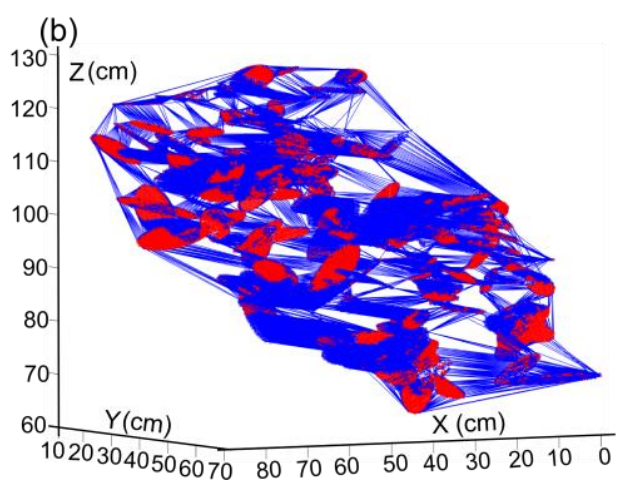

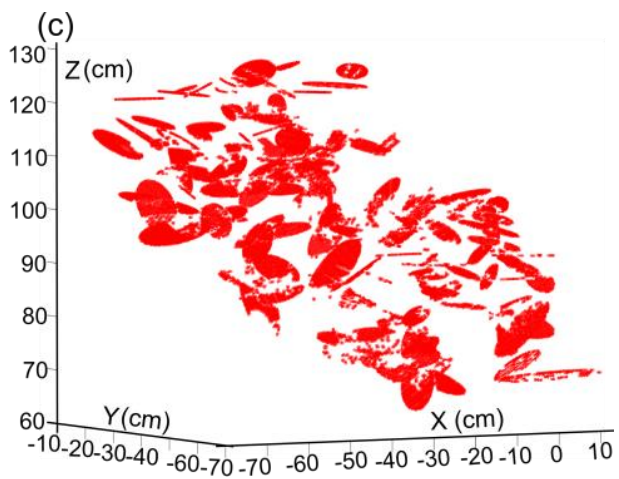

Fig. S4. Leaf surface reconstruction from point clouds via Delaunay triangulation. (a) Reconstruction of a single leaf surface; red triangles are the scanned region, while blue triangles (perimeter greater than the threshold $\zeta$ ) represent occluded areas and gaps between leaves; (b) section of a tree crown indicating blue triangles spanning adjacent virtual leaves, which are excluded in the filtering step; (c) remaining triangles representing the leaf area covered by laser beams are used for scanned leaf area assessment. 
3D triangulation for a large number of scanned points is time consuming and complex. Various orthographic projection strategies were proposed here to optimize the $3 \mathrm{D}$ triangulation process and to reduce the complexity of the algorithm. Various projection modes of the scanned points onto the $\mathrm{X}-\mathrm{Y}$ plane, $\mathrm{X}-\mathrm{Z}$ plane and $\mathrm{Y}-\mathrm{Z}$ plane were adopted. The projection mode corresponding to the minimal degree of the scanned points overlapping each other was selected as the preferred mode. Then, the 2D Delaunay triangulation algorithm was applied to create triangle meshes based on these projected points on the projection plane and the point indices defining each triangle that make up the triangulation were recorded. According to these point indices, a reverse projection from the $2 \mathrm{D}$ projection plane to the $3 \mathrm{D}$ space was operated to realize the 3D Delaunay triangulation for the scanned data. 